\title{
Clinical Effect of Lateral Transforaminal Endoscopic Surgery in the Treatment of Lumbar Disc Herniation
}

\author{
Weiya Zhang* \\ Yancheng No.1 People’s Hospital, Yancheng 224700, Jiangsu Province, China \\ *Corresponding author: Weiya Zhang, 1184259954@qq.com
}

\begin{abstract}
Objective: To analyze the efficacy of lateral transforaminal endoscopic surgery in the treatment of lumbar disc herniation. Methods: A total of 44 patients with lumbar disc herniation, treated in Yancheng No.1 People's Hospital from January 2019 to June 2020, were randomly divided into two groups; the 24 patients in group A were treated by lateral transforaminal endoscopic surgery, while the 20 patients in group B were treated by routine lamina fenestration and nucleus pulposus surgery. Results: The curative effect of group A $(91.67 \%)$ was higher than that of group B $(85.0 \%), P>0.05$; there was no significant difference in the angle from straight leg raise between group A and group B $(P>0.05)$; the Oswestry Disability Index (ODI) and the visual analogue scale (VAS) of group A were lower than those of group B $(P<0.05)$; the incision length, intraoperative blood loss, and hospital stay of the patients in group A were better than those in group B, $P<$ 0.05. Conclusion: Lateral transforaminal endoscopic surgery can significantly reduce pain, improve symptoms, and help to restore normal function in the early stage for patients with lumbar disc herniation.
\end{abstract}

Keywords: Lumbar disc herniation; Lateral transforaminal endoscopic surgery; Curative effect

Publication date: November 2021; Online publication: November 30, 2021

\section{Introduction}

Lumbar disc herniation refers to a partial or complete rupture of the fibrous ring upon degeneration of the lumbar disc, resulting in the protrusion of nucleus pulposus, which can compress the cauda equina and nerve roots, thus inducing a series of syndromes. In recent years, the lifestyle of Chinese people has become more and more diversified, and the incidence of lumbar disc herniation is increasing year by year. It has developed into a common spinal surgical lesion, which can cause low back pain, lower limb pain, and numbness ${ }^{[1]}$. At present, the treatment of this condition is still largely by surgical means, in order to restore spinal function, improve patients' quality of life, and reduce pain. Lateral transforaminal endoscopic surgery is a minimally invasive surgery, with little damage to patients and low risk of postoperative complications, which is more conducive to patients' postoperative rehabilitation. In addition, the application of intervertebral foramen mirror can also expand the surgical vision of surgeons as well as ensure the safe and smooth completion of the surgery ${ }^{[2]}$. Based on the treatment of 44 patients with lumbar disc herniation, this research explores the curative effect of lateral transforaminal endoscopic surgery.

\section{Materials and methods}

\subsection{General information}

A total of 44 patients with lumbar disc herniation, treated in Yancheng No.1 People's Hospital from January 2019 to June 2020, were randomly divided into two groups. In group A, the male to female ratio was 9:15, 
age ranging from 38 to 70 years old, with a mean age of $60.22 \pm 3.86$; the course of illness was 2-13 months, with a mean of $6.85 \pm 0.62$ months. In group B, the male to female ratio was $7: 13$, age ranging from 42 to 68 years old, with a mean age of 57.69 \pm 3.09 ; the course of illness was 3-20 months, with a mean of $8.11 \pm 1.04$ months.

\subsection{Inclusion and exclusion criteria}

The inclusion criteria were as follows: (1) symptoms and signs consistent with the auxiliary examination (CT and MRI); (2) a diagnosis of lumbar disc herniation via CT and MRI, all of which are single segment and unilateral herniation of L4/5 or L5/S1; (3) those who failed conservative treatment; (4) those who had given informed consent to different surgical methods; (5) those without any history of lumbar spine surgery. The exclusion criteria were as follows: (1) those with liver and kidney dysfunction; (2) those with contraindications to surgery; (3) those with immune dysfunction; (4) those with coagulation dysfunction; (5) those with multi-segment lumbar disc herniation, lumbar spondylolisthesis, and high iliac crest. The sample data of lumbar disc herniation in the two groups were compared $(P>0.05)$.

\subsection{Methods}

The patients in group A were treated with lateral transforaminal endoscopic surgery. The patients were placed in a prone position. The needle route was determined based on the size of intervertebral foramen, body shape, intervertebral disc position, and other factors. The puncture was made under the guidance of G-arm imaging, and $0.5 \%$ lidocaine was injected into the base of the upper articular process from shallow to deep for local anesthesia. An 18G puncture needle was prepared to enter the safe triangle of the intervertebral foramen through the perspective of the predetermined needle entry point until the intervertebral disc. The $18 \mathrm{G}$ needle core was then pulled out, and a $22 \mathrm{G}$ needle was inserted; $1 \mathrm{ml}$ of methylene blue was injected into the intervertebral disc to dye the nucleus pulposus. The $22 \mathrm{G}$ needle was then removed, and a guide wire was inserted. The $18 \mathrm{G}$ needle tube was removed, and the skin was cut about $1 \mathrm{~cm}$ in length; then, a guide rod was inserted along the guide wire. The soft tissues were expanded, and a cannula was placed in. The guide rod was then removed, and a circular saw was inserted; the ventral base of the superior articular process and the intervertebral foramen were expanded. The gap and position were confirmed under fluoroscopy. The circular saw was removed, and the intervertebral foramen mirror was connected to properly separate and carry out electrocoagulation under the direct vision of the screen. Upon visualizing the breach in the fibrous ring, the free nucleus pulposus was taken out, and the free nucleus pulposus tissue in the disc was cleaned. The nerve root activity and dural pulsation were checked. The fibrous breach was repaired, and the incision was sutured. During the surgery, the reaction of the patient should be observed to determine whether the nerve root had been touched to prevent nerve root injury. The whole surgery required direct vision to identify the nerve roots, dural sac, and stained intervertebral disc. Anti-infective drugs were given after the surgery.

The patients in group B underwent conventional posterior laminectomy to extract the nucleus pulposus. General anesthesia with endotracheal intubation were initiated. The patient was placed in a prone position. Using the G-arm to locate, the operation site was determined. Routine disinfection and the laying of sheets were done. An incision of about $8 \mathrm{~cm}$ long was made along the spinous process, cutting layer by layer and properly separating the erector spinalis muscle, exposing the affected side lamina space to the outer edge of the articular process. Paying attention to electrocoagulation and hemostasis, lamina biting forceps were used to bite off part of the bone at the lower edge of the lamina and the inner edge of the upper and lower articular processes. The ligamentum flavum was carefully separated, the dural sac and lateral nerve root 
were exposed, and the nerve root was pulled inside with the nerve retractor to clearly demarcate the prominent nucleus pulposus tissue. In order to protect the dural sac and nerve root, bipolar electrocoagulation was done properly, the prominent fibrous ring was cut with a sharp knife, the fragments of nucleus pulposus and the nucleus pulposus in the disc were taken out, the relaxation of nerve root and pulsation of dural sac were inspected, and the incision was washed. A drainage tube was placed, and the incision was sutured layer by layer. Anti-infective drugs were given after the surgery.

\subsection{Observation indexes}

If the discomfort symptoms at the lumbar spine and lower limbs disappear along with the recovery of function, it is recorded as remarkably effective; if the discomfort symptoms at the lumbar spine and lower limbs improve and the function basically recovers, it is recorded as effective; if there is no remission of the discomfort symptoms at the lumbar spine and lower limbs as well as recovery to the function, it is recorded as ineffective ${ }^{[3]}$.

\subsection{Statistical analysis}

The data of the patients with lumbar disc herniation were processed using SPSS 21.0. The sample data were recorded in percentage and $\bar{x} \pm \mathrm{s}$. The difference between the two groups was tested using t-test (t) and chisquare $\left(\mathrm{X}^{2}\right) . P<0.05$ indicates a significant difference.

\section{Results}

\subsection{Curative effect}

The curative effect of group A was $91.67 \%$, which was higher than that of group B $(85.00 \%), P>0.05$ (Table 1).

Table 1. Curative effect of the two groups (n/\%)

\begin{tabular}{ccccc}
\hline Group & Remarkably effective & Effective & Ineffective & Total effective rate \\
\hline Group A $(\mathrm{n}=24)$ & $17(70.83)$ & $5(20.83)$ & $2(8.33)$ & 91.67 \\
Group B $(\mathrm{n}=20)$ & $12(60.00)$ & $5(25.00)$ & $3(15.00)$ & 85.00 \\
$\mathrm{X}^{2}$ & - & - & - & 0.3332 \\
$P$ & - & - & - & $P>0.05$ \\
\hline
\end{tabular}

\subsection{Angle in straight leg raise, ODI, and VAS}

There was no significant difference in the angle in straight leg raise between group A and group B, $P>$ 0.05. The ODI and VAS of group A were lower than those of group B, $P<0.05$ (Table 2).

Table 2. Angle in straight leg raise, ODI, and VAS in the two groups $(\bar{x} \pm \mathrm{s})$

\begin{tabular}{ccccccc}
\hline Group & \multicolumn{2}{c}{ Angle in straight leg raise $\left({ }^{\circ}\right)$} & \multicolumn{2}{c}{ VAS (points) } & \multicolumn{2}{c}{ ODI (\%) } \\
& Preoperative & Postoperative & Preoperative & Postoperative & Preoperative & Postoperative \\
\hline Group A $(\mathrm{n}=24)$ & $36.52 \pm 2.82$ & $76.84 \pm 4.78$ & $7.35 \pm 0.82$ & $2.09 \pm 0.51$ & $46.11 \pm 5.24$ & $13.85 \pm 1.82$ \\
Group B $(\mathrm{n}=20)$ & $36.49 \pm 2.79$ & $75.46 \pm 3.24$ & $6.87 \pm 0.79$ & $4.18 \pm 0.79$ & $46.17 \pm 5.17$ & $25.46 \pm 2.03$ \\
$\mathrm{t}$ & 0.0353 & 1.0971 & 0.0762 & 10.5761 & 0.0355 & 19.0040 \\
$P$ & $>0.05$ & $>0.05$ & $>0.05$ & $<0.05$ & $>0.05$ & $<0.05$ \\
\hline
\end{tabular}




\subsection{Surgical indexes}

The incision length, intraoperative bleeding, and hospital stay of the patients in group A were better than those of group B $(P<0.05)$. There was no significant difference in the operating time between group A and group B $(P>0.05)$ (Table 3).

Table 3. Surgical indexes of the two groups (n/\%)

\begin{tabular}{ccccc}
\hline Group & Operating time (minutes) & Incision length $(\mathrm{cm})$ & $\begin{array}{c}\text { Intraoperative bleeding } \\
\text { volume }(\mathrm{ml})\end{array}$ & Hospital stay (days) \\
\hline Group A $(\mathrm{n}=24)$ & $80.13 \pm 2.46$ & $0.93 \pm 0.34$ & $19.33 \pm 3.36$ & $6.19 \pm 1.46$ \\
Group B $(\mathrm{n}=20)$ & $78.38 \pm 3.38$ & $8.48 \pm 1.06$ & $88.43 \pm 5.17$ & $11.44 \pm 2.78$ \\
$\mathrm{t}$ & 1.9846 & 20.0802 & 30.1362 & 6.6799 \\
$P$ & $>0.05$ & $<0.05$ & $<0.05$ & $<0.05$ \\
\hline
\end{tabular}

\section{Discussion}

The pathological basis of lumbar disc herniation is disc degeneration, which is induced by cumulative disc injury. The pathogenesis of lumbar disc herniation is related to long-term high load state of the lumbar disc, resulting in the tearing of annulus fibrosus. Under the influence of trauma, the diseased nucleus pulposus prolapses through the fissure in the annulus fibrosus, resulting in nerve compression and various diseases ${ }^{[4]}$. At present, the clinical treatment of lumbar disc herniation includes conservative treatment and surgery to alleviate the symptoms of the herniated lumbar disc ${ }^{[5]}$. Conservative treatment involves the positional change of intervertebral disc tissues and compressed nerve roots to reduce nerve root compression and relieve pain. Common conservative treatment options include massage, traction, bed rest, epidural injection of corticosteroids, etc. ${ }^{[6]}$. However, for those with dyskinesia, severe pain, or ineffective conservative treatment, surgery is recommended. The commonly used surgical methods include posterior laminae fenestration of nucleus pulposus, lateral transforaminal endoscopy, discectomy, and bone graft fusion with internal fixation. Lateral transforaminal surgery can reduce surgical trauma, improve postoperative adhesion, expand the surgical field under endoscopic surgery, accurately remove pathological tissues, and reduce the impact on spinal stability ${ }^{[7]}$. Lateral transforaminal endoscopic surgery has several advantages.

(1) Its small surgical incision reduces the pain experienced by patients. The lateral approach reduces the injury to the ligamentum flavum of the lamina and the stability of the intervertebral plate. It is not necessary to bite off the lamina during the surgery; hence, it will not damage the paravertebral muscles and ligaments, thus having a low impact on the stability of the spine.

(2) The surgery has high safety. Only local anesthesia is used; hence, patients would be awake during the surgery. There is minimal intraoperative bleeding, and the surgical field is clear, thus reducing the risk of errors in surgery.

(3) It is conducive to the rehabilitation of patients, reduces the risk of postoperative complications, shortens the time for mobilization, and reduces the length of hospital stay ${ }^{[8]}$. However, during the actual procedure, it is necessary to pay attention to several points. First, the lateral approach can directly enter the ventral side of the articular process. Under the guidance of fluoroscopy, the guide wire and catheter can be correctly used to completely remove the intervertebral disc tissue at the spinal canal, intervertebral foramen, and other positions. Second, it is necessary to clarify the indications for the surgery. Lateral foraminal endoscopic surgery can be used in patients with nerve root compression caused by paracentral and extreme lateral disc herniation ${ }^{[5]}$. 
Based on the data analysis above, the postoperative curative effect of group A (91.67\%) was higher than that of group B $(85.00 \%)$; the ODI and VAS of group A were lower than those of group B; the incision length, intraoperative bleeding, and hospital stay of the patients in group A were better than those of group $\mathrm{B}$, suggesting that lateral foraminal endoscopic surgery is of great value in the treatment of lumbar disc herniation. There were no complications, such as nerve root injury, surgical site infection, as well as spinal canal and intervertebral space infection in both groups.

In conclusion, with appropriate indications, patients with lumbar disc herniation can be treated with lateral transforaminal endoscopic surgery. It can significantly reduce patients' pain, improve symptoms, facilitate early functional recovery, and restore their ability for normal activity as soon as possible.

\section{Disclosure statement}

The author declares that there is no conflict of interest.

\section{References}

[1] Cui H, Lin H, 2020, Analysis on the Clinical Effect of Spinal Foraminal Endoscopy in the Treatment of Patients with Protrusion of Lumbar Intervertebral Disc. Journal of Clinical Medical Literature, 445(24): 61.

[2] Shi J, Li Yu, Li H, et al., 2018, Clinical Observation of Spinal Foraminal Endoscopic Surgery in the Treatment of Protrusion of Lumbar Intervertebral Disc. World Latest Medicine Information, 18(88): 187-188.

[3] Wang D, Ji B, Zhang D, et al., 2017, Effect Analysis of Targeted TESSYS Foraminoscopy in the Treatment of Protrusion of Lumbar Intervertebral Disc. Orthopedic Journal of China, 25(425): 92-95.

[4] Yan H, 2017, Clinical Effect of Percutaneous Foraminoscopy for Spinal System Technique in the Treatment of Lumbar Disc Herniation. Global Traditional Chinese Medicine, 10(018): 137-138.

[5] Xu L, Xu W, Liu Y, et al., 2018, Clinical Effect Comparation of Microincision Microsurgery and Spinal Foraminal Endoscopic Surgery in the Treatment of Protrusion of Lumbar Intervertebral Disc. Aerospace Medicine, 029(002): 158-160.

[6] Fang M, Huang K, 2020, Comparison of Percutaneous Foraminoscopy and Open Surgery for Patients with Protrusion of Lumbar Intervertebral Disc. Guide of China Medicine, 18(31): 80-81.

[7] Pan D, Zheng X, Wang L, 2019, Clinical Effect and Value of Spinal Foraminal Endoscopic Surgery in the Treatment of Protrusion of Lumbar Intervertebral Disc. Clinical Research, 27(8): 3.

[8] Zhang D, Zhou J, Wu J, et al., 2017, Comparison of Clinical Effects of Percutaneous Foraminal Endoscopy and Laminectomy in the Treatment of Recurrent Lumbar Disc Herniation. Chinese Journal of New Clinical Medicine, 07(22): 43-45. 